\title{
Industrial Wireless End-to-End Measurements and Impacts in a Gas-Sensing Scenario
}

\author{
Mohamed Kashef and Richard Candell \\ National Institute of Standards and Technology, \\ Gaithersburg, MD 20899, USA \\ mohamed.kashef@nist.gov \\ richard.candell@nist.gov
}

\begin{abstract}
Industrial wireless is a potential networking solution in many scenarios due to its flexibility and ease of communications in harsh environments. Industrial wireless in gas-sensing and air-quality monitoring applications is essential when wired communications cannot perform the task safely and effectively. A major example of such environments is confined spaces where attaching mobile gas sensors with wires is a major concern for safety and cannot be deployed in some cases. At the National Institute of Standards and Technology (NIST), we developed an end-to-end characterization method for industrial wireless networks. We employed this characterization method to study the end-to-end error and delay performance for a confined-space gas-sensing scenario. We have built the scenario using the NIST industrial wireless test bed, which includes ISA100.11a wireless devices, a channel emulator, and a high-performance programmable logic controller (PLC), where the physical process is simulated. In this work, we studied the effects of the size of the confined space, the relaying, input signal rate, and the impact of the existing workers in the confined space.
\end{abstract}

Key words: industrial wireless; manufacturing; networked control; process control.

Accepted: November 20, 2018

Published: December 11, 2018

https://doi.org/10.6028/jres.123.023

\section{Introduction}

\subsection{Industrial Wireless Applications}

The Industrial Internet of Things (IIoT) aims to achieve lower deployment costs, increased efficiency of operation, and improved mobility of the industrial environment. Industrial wireless is a key technology for IIoT. Wired solutions require expensive cables, conduits, and labor to install, making wired solutions uneconomical, and in many cases environmental conditions make installation of cables impractical. Hence, wireless solutions may be advantageous to manufacturing operations [1].

Industrial wireless can be deployed in various use cases with different constraints and different requirements. The problem space for industrial wireless has been categorized in Ref. [2] to the following application areas: instrumentation, personnel safety, back-haul connectivity, tracking, surveillance, remote assets, and maintenance support. For each of these areas, different wireless technologies could be deployed. The applicability of various technologies to the corresponding application areas has been discussed in many articles, such as Refs. [2-4]. 
Industrial settings can be hazardous to both humans and machines. For humans, conditions may arise that pose a substantial risk for injury or death. For machines, conditions may develop that cause substantial damage, requiring extensive repair or replacement. Prevention of industrial accidents is therefore of paramount importance within factories [5]. Within the oil and gas industry, safety concerns include air toxicity and combustibility in both open and confined spaces, where reliable monitoring and reporting can save lives. Wireless gas leak detection and leak localization provide important and effective safety enhancements to such systems [6]. Within smart manufacturing systems, where humans and robots work closely together, and even within traditional robot environments, safety systems provide an added layer of protection to prevent human injury [7, 8]. Within these human-robot environments, it is clear that reliable, low-latency communication is an important aspect of safety implementation, and, as mobility of robots within factories increases, reliable low-latency wireless networks will become increasingly important to safety implementation.

\subsection{Gas Sensing in Confined Spaces}

Gas sensing has become a typical component in many industrial systems because of its broad usage in many industrial areas, such as manufacturing, automotive, and medical industries. Specifically, gas leak monitoring is employed for safety purposes in scenarios when leaks can result in human fatalities. Moreover, distributed gas-sensing networks are widely deployed because of different gas densities at different locations and heights. Hence, wiring of these distributed networks represents a major concern in implementation because of the costs and complexity of wiring in some environments. As a result, wireless gas-sensing networks provide a more flexible and suitable solution for continuous distributed gas-sensing applications.

Moreover, confined-space monitoring plays an important role in reducing injury that may happen due to gas accumulations. Entering a confined space to perform a maintenance task requires following a proper safety protocol. The major requirements of safety protocols typically include checking air quality before entering and having a portable gas sensor for continuous gas-level monitoring while inside. Hence, technologies of gas monitoring equipment are being advanced to meet these requirements. Wireless communication is used to connect distributed gas monitoring sensors to various control and alarming units allowing continuous monitoring and reliable measurements from portable devices [9].

\subsection{Purpose and Approach}

Wireless communications help to alleviate cost and agility constraints by being low-cost and mobile [1]. However, industrial wireless environments can be harsh, and the surroundings are typically highly metallic, thereby creating multipath effects and rapid path loss. Industrial control systems often require very high transmission reliability [10]. Depending on the purpose of the wireless network (monitoring, supervisory control, feedback control, or safety monitoring), understanding the system performance of the network may be critical. Factory operators, system integrators, and control systems designers are rarely experts in wireless communications systems. Considerations such as electromagnetic propagation, antenna efficiency, path loss exponents, packet error rates, and medium access are often foreign concepts to factory engineers. Moreover, it is generally difficult to measure these quantities for operational networks. The control system design will only need to know the statistical distribution of latency and reliability of information throughout the network to design a controller that is robust. Therefore, a practical method for characterizing the performance of the wireless network that does not require an in-depth understanding of wireless communications or electromagnetic wave propagation is important.

The primary objective of this work was to evaluate the performance of a wireless network deployed in an industrial setting. The end-to-end performance was characterized in order to study the effects of various 
wireless networking aspects on the gas-sensing safety requirements. In this work, we tested the applicability of using wireless sensing in gas-level detection in confined spaces. We focused on the use of ISA100.11a wireless nodes ${ }^{1}$ to transmit a typical accumulating carbon monoxide (CO) gas concentration signal. We characterized the performance of the deployed wireless system by calculating the end-to-end delay and error in the transmitted signals. We also used a channel emulator to generate confined-space wireless channel effects on the signals. We studied the effects of various system parameters on the performance to generally assess the use of wireless communications in confined spaces for gas monitoring.

\subsection{Previous Work}

The problem of studying the performance of industrial wireless networks has been approached from different viewpoints previously. First, simulations of industrial wireless networks have been conducted to study various performance measures, such as in Refs. [1, 11-14]. Second, the performance has been measured using hardware experiments, as in Refs. [15-22].

In simulation-based performance analyses, the packet-level measures are easily monitored throughout the simulation. In Ref. [1], a simulation framework was introduced for using a WirelessHART communication network in a process control system where all packet-level parameters were controlled and monitored. In Refs. [11, 12], the use of various simulation packages for simulating wireless networks in cyber-physical systems was considered. Moreover, in Ref. [13], an ISA100.11a system was studied where the effects of various network parameters were considered by evaluating packet-level measures, such as throughput, average delay, and energy consumption. Finally, in Ref. [14], the reliability of an industrial wireless network was studied by measuring the communication latency and stability on the packet level.

On the other hand, hardware analyzing or testing tools can be used to monitor packet-level measures [15], while network performance can be studied for compatible hardware experiments. In Refs. [16, 17], a self-developed wireless network was used to monitor a turbine power-generation system. In Refs. [18, 19], IEEE 802.15.4a development boards were used as the network devices, where configuration and monitoring could be easily done through an attached computer. Similarly, in Ref. [20], single-hop transmissions were evaluated using accessible hardware where packets were controlled. Also, in Ref. [21], wireless stations that were compliant with both IEEE 802.11g and IEEE 802.11e specifications were used for wireless communications, where the response time was measured in a four-node network. Finally, in Ref. [22], Zigbee programmable nodes were used in communications.

In Refs. [23-27], the use of wireless gas sensing was introduced in various scenarios. In Ref. [23], a wireless gas sensor was introduced as an example of the smart-sensing applications, where the sensor is able to sense various types of gases, store data, and generate alarms through the integration of a microprocessor. In Ref.[24], a long-term air-quality monitoring test bed was built and evaluated. A wireless IEEE 802.15.4-based node was continuously operated and it was compared to a standard non-wireless air-quality monitoring system. In Ref. [25], an experimental setup of a sensor-actuator system for gas detection and control was introduced. The system used wireless sensors and actuators that deployed the Zigbee standard with the BACnet building automation protocol. In Ref. [26], the use of wireless gas sensing was discussed in industrial environments. Those authors justified the importance of wireless gas-sensing networks due to the ease of installation and the low cost of maintenance. Finally, in Ref. [27], the use of wireless gas sensing was proposed for underground gold and platinum mines to improve gas detection capabilities, because the existence of methane gas in these mines can cause fires and toxicity.

${ }^{1}$ Certain commercial equipment, instruments, or materials are identified in this paper in order to specify the experimental procedure adequately. Such identification is not intended to imply recommendation or endorsement by the National Institute of Standards and Technology, nor does it imply that the materials or equipment identified are necessarily the best available for the purpose. 


\subsection{Contributions and Organization}

Our contributions in this work can be summarized as follows:

- We tested and analyzed the performance of a commercial ISA100.11a network as an example of industrial wireless technologies in a gas-sensing safety application. We employed a hardware-in-loop (HIL) simulator for the studied scenario, including a channel emulator to capture the confined-space channel impact.

- We characterized the end-to-end performance of the wireless network by studying the error, delay, and the input-output cross-correlation. We discuss the applicability of the industrial wireless solution at various system settings.

- We assessed the effects of different network parameters including relaying, the input signal rate, the size of the confined space, the type of input signal, and the existence of workers in the confined space.

The rest of the paper is organized as follows. In Sec. 2, we describe the general system model, the testbed used for the study, and the tested channel models. In Sec. 3, we detail the proposed test method and the various performance criteria used. Then, in Sec. 4, we discuss the experimental results and industrial network characterization. In Sec. 5, we draw some conclusions.

\section{System Model}

\subsection{Physical System Model}

In this study, we considered the case of welding a tank where the welding process produces gases in rates that are generally nonhazardous. In confined spaces, gas accumulations may lead to an increased concentration level of these gases, which may lead to high-risk situations [28, 29]. Specifically, the welding smoke can be extremely toxic, because it contains many substances, such as chromium, nickel, arsenic, asbestos, manganese, silica, beryllium, cadmium, nitrogen oxides, phosgene, acrolein, fluorine compounds, carbon monoxide, cobalt, copper, lead, ozone, selenium, and zinc. A case study in which CO became dangerous and life-threatening was discussed in Ref. [30].

The existence of $\mathrm{CO}$ at high concentrations can lead to poisoning of humans and increased risk of fire and explosion. For a gas to lead to a fire or an explosion, it has to be in the range between the lower explosive limit (LEL) and the upper explosive limit (UEL), which are $12.5 \%$ and $74 \%$ of the volume, respectively, for CO. Moreover, the permissible exposure limit (PEL) of CO is 25 parts per million (ppm), which defines a threshold for alarming a worker to vacate from the confined space.

The considered scenario is shown in Fig. 1. There is a cylindrical tank with a diameter $H$ and a length $X$, and another cylindrical manhole with height $D$ connects the tank to the outer open space. An ISA100.11a gas sensor is attached at the welding position (A) to measure the CO level at this point. Another ISA100.11a gas sensor is positioned at the manhole end at the tank side (B). The ISA100.11a access point (AP) is positioned at the other end of the manhole (C) to have a line-of-sight (LOS) channel with the ISA100.11a node at $\mathrm{B}$.

\subsection{Simulation Architecture}

Performance evaluation was conducted in an industrial wireless continuous process test bed. The test bed is a reconfigurable platform that was designed to evaluate the effects of wireless communications on a continuous process system. The test bed includes a high-performance programmable logic controller (PLC), where signals are produced. These signals represent typical signals in a certain industrial plant. As an 


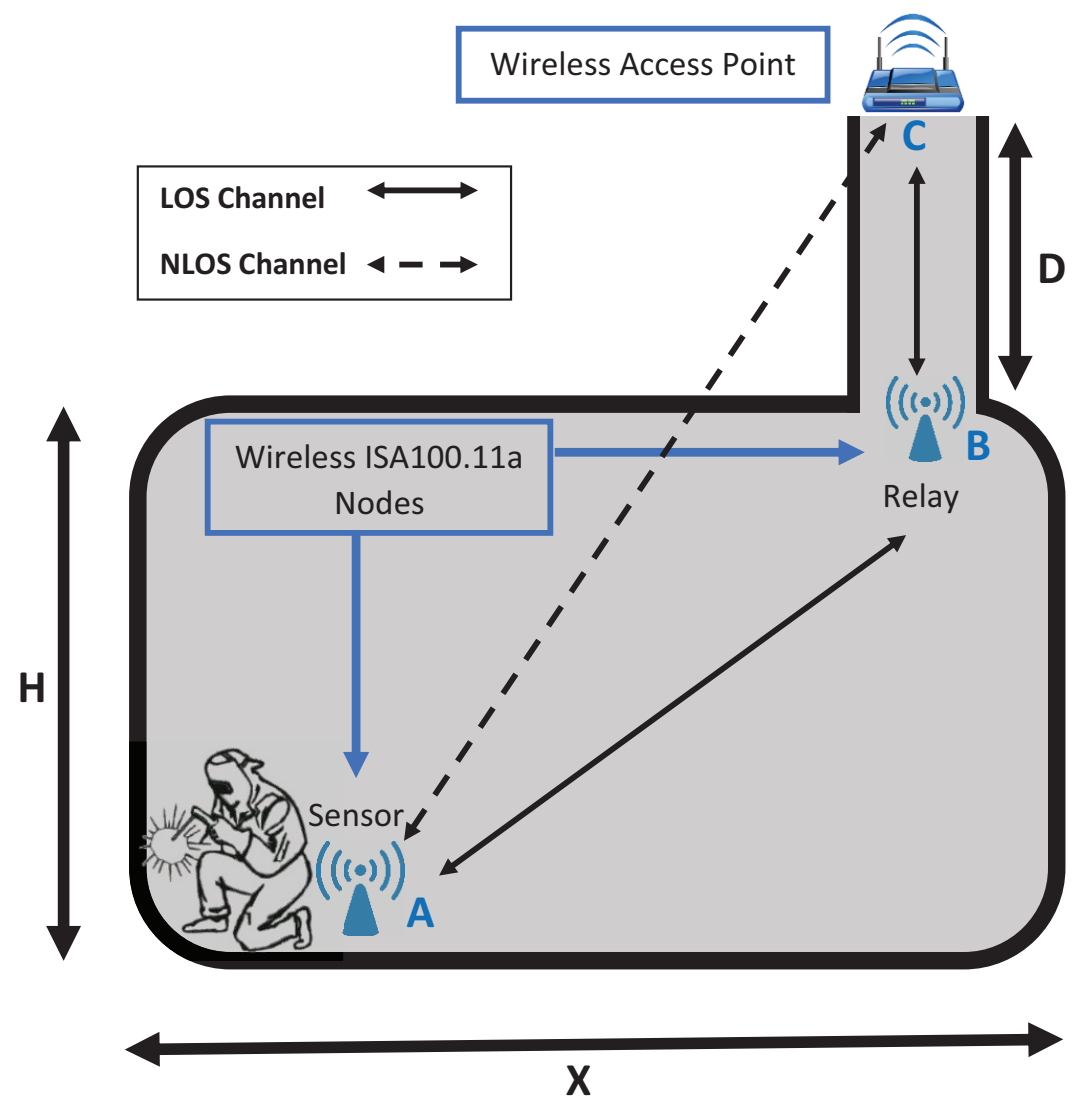

Fig. 1. Physical system model where LOS stands for line of sight and NLOS stands for non LOS.

example, gas accumulations are represented by a charging exponential signal or a ramp signal. The PLC is equipped with 16 bit digital-to-analog (D/A) conversion module.

The wireless network is composed of wireless ISA100.11a sensors and an AP. In the test bed, we employed a channel emulator in order to include industrial environment effects in wireless transmissions. A radio frequency channel emulator was used that is capable of replicating the multi-path and path loss environment for a mesh network of up to 8 physical nodes and 56 virtual links between those nodes. The channel emulator was an RFnest D508, and the corresponding software is RFview [31]. The channel emulator supports an instantaneous bandwidth of $250 \mathrm{MHz}$ (4 ns tap spacing) with an effective dynamic range of $73 \mathrm{~dB}$, which includes all analog and digital realization impacts. The emulator is controlled by a nearby computer, which loads the path loss model and channel impulse response for each communications link. The transmitted signal is received by a wireless gateway.

\subsection{Channel Models and Integration}

Generally, industrial wireless environments can be harsh, because the surroundings are typically highly reflective, thereby creating significant multipath effects. Hence, it is important to deploy testing over the industrial wireless channels. In this subsection, we briefly describe the channel models that were considered in this work. We also describe the application of these models using the channel emulator.

Based on node positions, LOS and non-LOS (NLOS) channels may exist. The links between the positions A-B and B-C have LOS channels, while the link A-C has a NLOS channel, except when $D=0$. 
LOS channels are typically characterized, compared to NLOS channels, by a lower loss exponent and a channel impulse response (CIR) with more energy on the first peak (higher K-factor).

First, we considered the benchmark channel with log-distance path loss and ideal CIR, which has no multipath. Second, we considered a measured delay profile of a confined space where the CIR was experimentally measured and processed using the channel emulator and to reflect the confined-space industrial channel impact. Both LOS and NLOS CIRs were measured in this case. While deploying the measured channels, the emulator produces random Rayleigh fading channels for the NLOS cases and random Rician fading channels for the LOS cases, following the injected CIRs. The ideal log-distance models have the same loss exponents as their counterpart measured channel models, but they have an ideal single-tap CIR.

Finally, the described channel models were applied using the channel emulator. The emulator implements a finite impulse response (FIR) filter at its core. The computational limitations of the channel emulator constrain the FIR filter and the channel CIR to 13 taps. In order to use the obtained CIR for channel emulators, we used the technique introduced in Ref. [32] to both resample and reduce the number of the taps of the CIRs within the limitations of the emulator.

\section{Test Method}

In this section, we describe the proposed testing approach in this work. The primary objective of this approach was to evaluate the performance of a wireless network deployed in an industrial environment. We assessed the end-to-end performance of the industrial wireless network resulting from injecting various types of input signals. Specifically as shown in Fig. 2, we injected a known input signal and monitored the output of the wireless network at the point where it is considered to be usable by another network node.

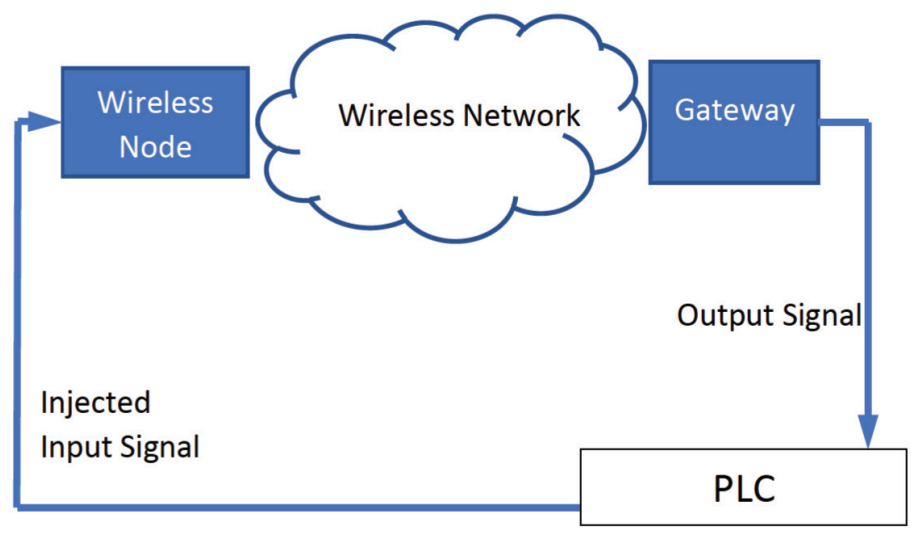

Fig. 2. The test method architecture in the case of wireless sensor node to gateway transmission.

In the following subsections, we discuss the various types of input signals that were introduced into the wireless network. We also discuss the considered analysis tools and performance criteria for characterizing a general industrial wireless network.

\subsection{Performance Criteria}

We denote the input signal to the tested wireless network by $x(t)$. This signal is an analog signal following the same characteristics of the electrical signals created by sensor nodes. The output signal is denoted by $y(t)$, which is a delayed and distorted version of the input signal. We assume that the input signal 
is delayed by $d(t)$, which is a time-varying delay because of the time-varying nature of the channel and various system components. Hence, the output signal is expressed as

$$
y(t)=x(t-d(t))+n(t)
$$

where $n(t)$ is a noise term that includes all the distortion effects in the wireless network, such as the additive white Gaussian noise (AWGN) of the channel and domain interfaces, the multipath delay spread, the interference by all in-network and out-of-network nodes, quantization error, and sampling distortion.

We define the variable $\tilde{d}$ as the end-to-end estimated average delay of the wireless network. The variable $\tilde{d}$ is optimized to get the optimal delay estimate, which minimizes the normalized average absolute error of the signal. This calculated optimal delay represents a time alignment metric.

The error value is denoted by $e(\tilde{d})$ and is evaluated using the absolute value of the difference between $y(t)$ and the delayed version of $x(t)$ as follows

$$
e(\tilde{d})=\frac{\int_{0}^{T}|y(t)-x(t-\tilde{d})| d t}{\int_{0}^{T}|x(t)| d t} .
$$

The optimal delay estimate, which is denoted by $\hat{d}$, is the value of $\tilde{d}$ that minimize the error function as follows

$$
\hat{d}=\underset{\tilde{d} \in[0, T]}{\arg \min } e(\tilde{d}),
$$

where $T$ is the testing period. This obtained delay, $\hat{d}$, represents the most accurate time alignment metric that captures the time-varying delay of the signal and also the alteration in the signal shape due to various distortions effects.

The value of error at $\hat{d}$ is the minimum obtained error at the output signal. It can be defined as follows

$$
\hat{e}=e(\hat{d})=\frac{\int_{0}^{T}|y(t)-x(t-\hat{d})| d t}{\int_{0}^{T}|x(t)| d t} .
$$

Various types of sources can cause errors at the transferred signals over industrial wireless networks. These sources include sampling, quantization, background noise, and interference.

Finally, the normalized cross-correlation $\beta$ is used to assess the general distortion of the signal, including the time-jitter and error effects. The cross-correlation function is obtained by integrating the lag product of the input and output signals over the testing period $T$. The cross-correlation function is denoted by $R(\tau)$ and is calculated as follows

$$
R(\tilde{d})=\frac{1}{T} \int_{0}^{T} y(t) x(t-\tilde{d}) d t
$$

The value of $\beta$ is evaluated by calculating the ratio between the peak of the $R(\tau)$ and the peak of the auto-correlation function of $x(t)$. This ratio is denoted by $\beta$ and is calculated as follows

$$
\beta=\frac{\max _{\tilde{d} \in[0, T]} R(\tilde{d})}{\frac{1}{T} \int_{0}^{T} x^{2}(t) d t} .
$$

The parameter $\beta$ takes values between 0 and 1 . When its value is closer to 1 , it indicates that the delay estimation is reliable, the variance in delay is low, and the amount of error in the received signal is small. 


\subsection{Input Signals}

In order to quantify the error effects on the performance of an industrial network, an arbitrary input signal is injected at the transmitting node. The injected signal is selected to be similar to practical signals of the sensing node, for example, being a linear signal with certain slope, or a signal with a charging behavior to represent gas accumulations. First, we consider a ramp signal where the concentration of the $\mathrm{CO}$ increases with a fixed rate over time. The slope of the signal at the sensor node is fixed but can have multiple values based on the welding process. The ramp signal saturates at $75 \mathrm{ppm}$. Then, we consider a charge signal to represent the effect of gas accumulations in a confined space where the concentration of the $\mathrm{CO}$ increases with a fixed time constant that can have multiple values based on the welding process. The time constant is smaller when the accumulation of the gas is faster at the sensor node. The end-to-end performance of these signals can be representative of the reliability of the network in the case of transmitting gas concentration signals from confined spaces.

\section{Results}

In this section, we illustrate the use of the proposed test method on the described testbed. We consider the scenario shown in Fig. 1 with various systems settings. We consider two sizes for the tank; $H=3.3 \mathrm{~m}$ for both of them, and $X$ takes the values $\{6,12\} \mathrm{m}$ where these two sizes are denoted by "Small" and "Big", respectively. The height of the manhole $D$ takes values of $\{0,1,2,3,4,5\} \mathrm{m}$. Two channel models are considered, the log-distance path loss channels with either ideal CIR or measured CIR, and they are denoted by "Log" and "Meas," respectively. The path loss exponents are 1.8 for LOS channels and 4.3 for the NLOS channels. Four different modes are considered: the relay does not exist, the relay exists with no humans inside the tank, the relay exists with one worker inside the tank, and the relay exists with two workers inside the tank. These modes are denoted by "Single," "Relay," "1 Human," and "2 Humans," respectively.

While running the simulations, the two types of input signals are denoted by "Linear" and "Charge." The linear signal takes the slope values of 1,5 , and $25 \mathrm{ppm} / \mathrm{min}$, and the corresponding results are labeled by "Slope-1," "Slope-2," and "Slope-3," respectively. The Charge signals take the time constants of 8.33, 1.67 , and $0.33 \mathrm{~min}$ for charging to the maximum of $75 \mathrm{ppm}$. The results are also labeled by "Slope-1," "Slope-2," and "Slope-3," respectively. Each of the simulations was run for $15 \mathrm{~min}$. The LOS channels are time varying with Ricean distribution, while the NLOS channels follow a Rayleigh distribution. Having a single worker in the confined space is modeled by adding $10 \mathrm{~dB}$ of attenuation to the received signal [33].

\subsection{Single Node Performance}

In this subsection, the performance of the case of point-to-point communications between the sensor node and the gateway is considered (Figs. 3-5). The performance degrades significantly with increasing manhole height due to the poor NLOS characteristics. The signal is almost lost at $D=5 \mathrm{~m}$ for the larger tank, linear signal, and measured CIR. Whenever the signal is lost, the values of the three evaluated performance metrics become very poor. As an example, the end-to-end delay has a very high value, which means that output and input cannot be aligned.

Generally, the performance of the more realistic measured CIR is worse than the corresponding ideal CIR with no multipath due to the signal energy spread in the time domain. Also, the larger is the tank, the worse is the performance. In Fig. 4, there is an initial delay difference of $4 \mathrm{~s}$ due to the size of the tank at $D=0 \mathrm{~m}$. The main source of this difference in the time alignment metric is due to the increase in the packets drop rate during the transmission and hence more signal distortion in the larger tank. The change of propagation delay in both tanks is minimal. 


\section{Journal of Research of National Institute of Standards and Technology}

The performance of the linear signal is worse than the charge signal because of the longer portion of the charge at which the signal has very low rate, and so the steady-state error affects the signal for a longer period. In conclusion, the performance of no relaying topology is satisfactory for smaller tanks.

Furthermore, the use of realistic CIR gives a better understanding of the system performance compared to the simple ideal CIR models.

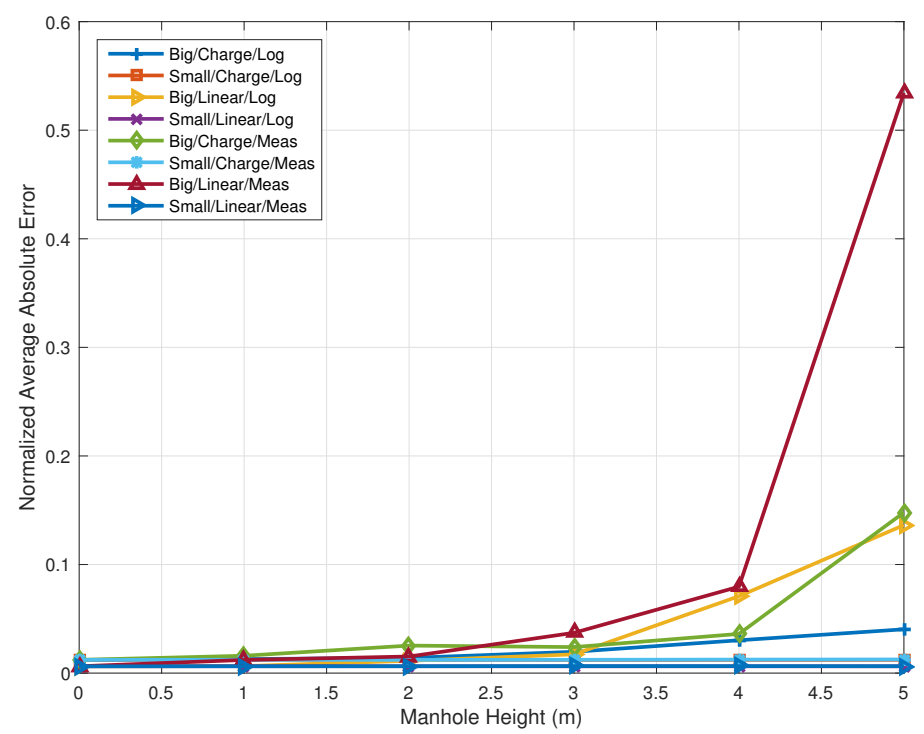

Fig. 3. Normalized absolute error for no relaying mode with linear signal at $5 \mathrm{ppm} / \mathrm{min}$ slope and charge signal with a time constant of $1.67 \mathrm{~min}$.

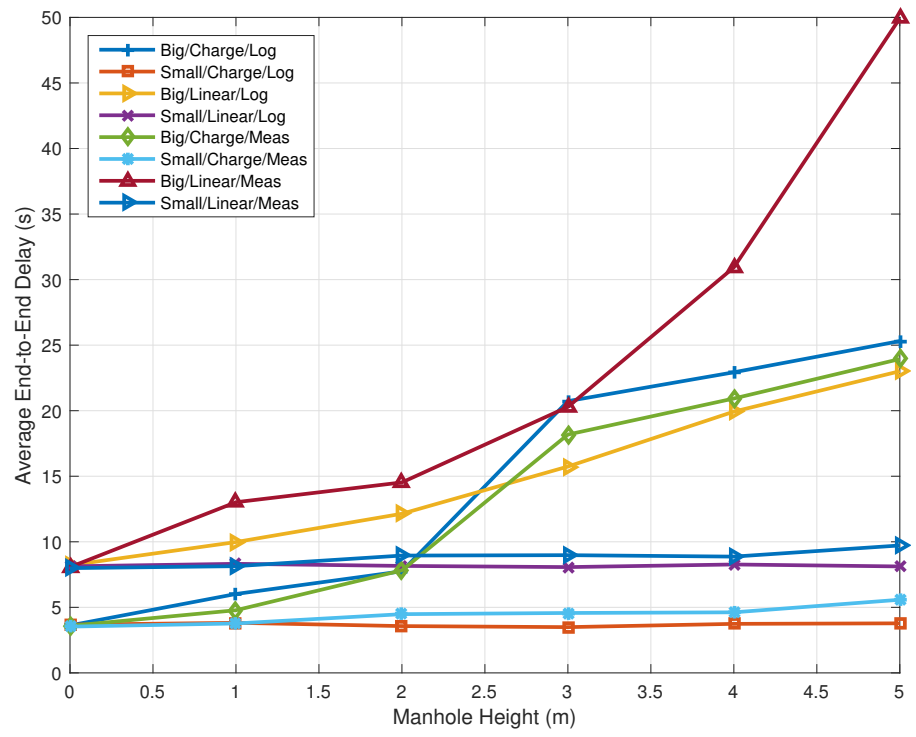

Fig. 4. End-to-end delay for no relaying mode with linear signal at $5 \mathrm{ppm} / \mathrm{min}$ slope and charge signal with a time constant of $1.67 \mathrm{~min}$. 


\section{Journal of Research of National Institute of Standards and Technology}

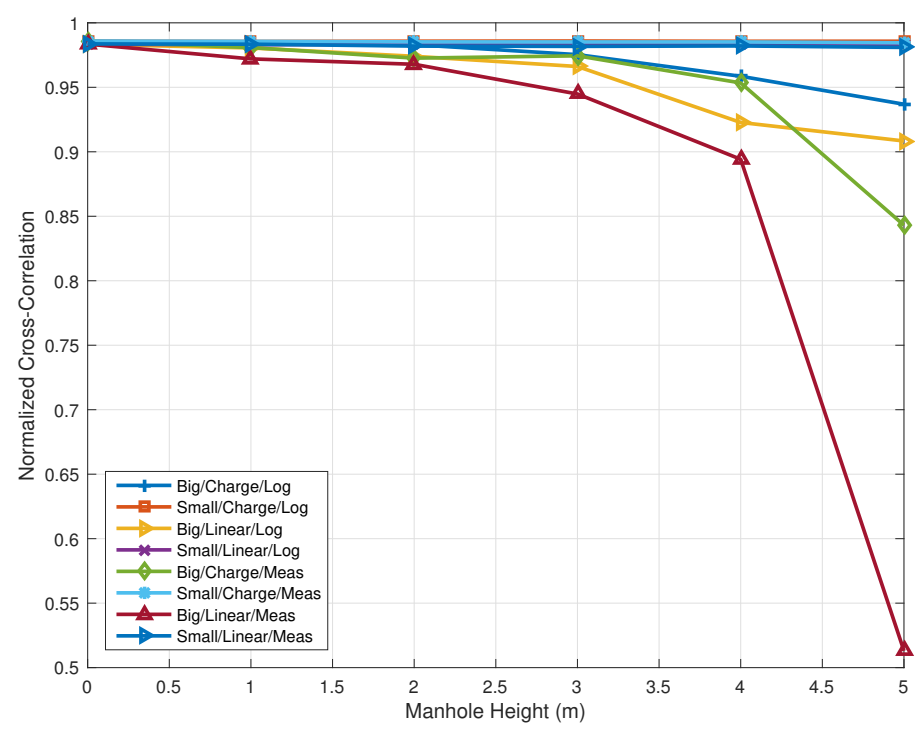

Fig. 5. Normalized cross-correlation for no relaying mode with linear signal at $5 \mathrm{ppm} / \mathrm{min}$ slope and charge signal with a time constant of $1.67 \mathrm{~min}$.

\subsection{Effect of Input Signal Rate}

In this subsection, we consider the effect of the input signal rate, which is represented by the slope of the linear signal or the time constant of the charge signal (Figs. 6-8). In the case of no relaying where the channel is poor, the increase of the linear signal slope increases the error significantly, as shown in Fig. 6. The same behavior can be found in the normalized cross-correlation in Fig. 8. On the other hand, the delay increases with decreasing slope, where slower signals have higher delay, as shown in Fig. 7. The error and cross-correlation increases with slope are due to the increased signal rate, where the missed part of the signals between sampling instants is larger. However, the time alignment metric is smaller due to the larger steady-state part of the input signal,

In Tables 1-3, the performance of the charge signal is shown for the big tank size. The delay performance is degraded significantly with manhole height, but error and cross-correlation are not similarly degraded. The results for the small tank size for both linear and charge signals are not shown for brevity; however, it was noticed that error and cross-correlation increased slightly with distance and with an increase of the input signal rate. The delay decreased with decrease of the input signal rate. 


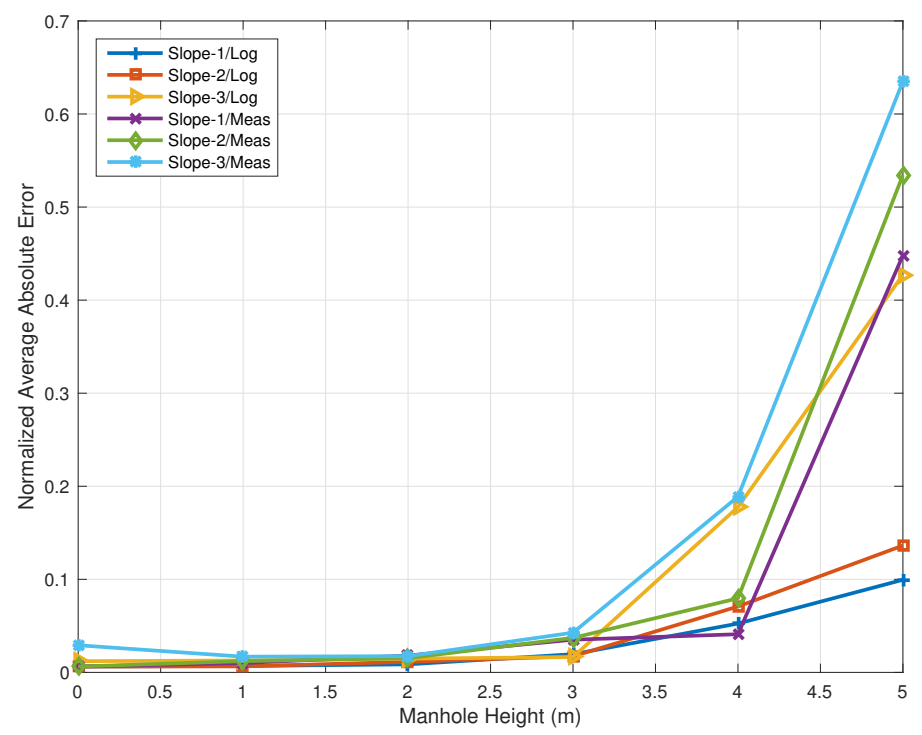

Fig. 6. Normalized absolute error for no relaying mode with big tank size and with linear signal at various slopes.

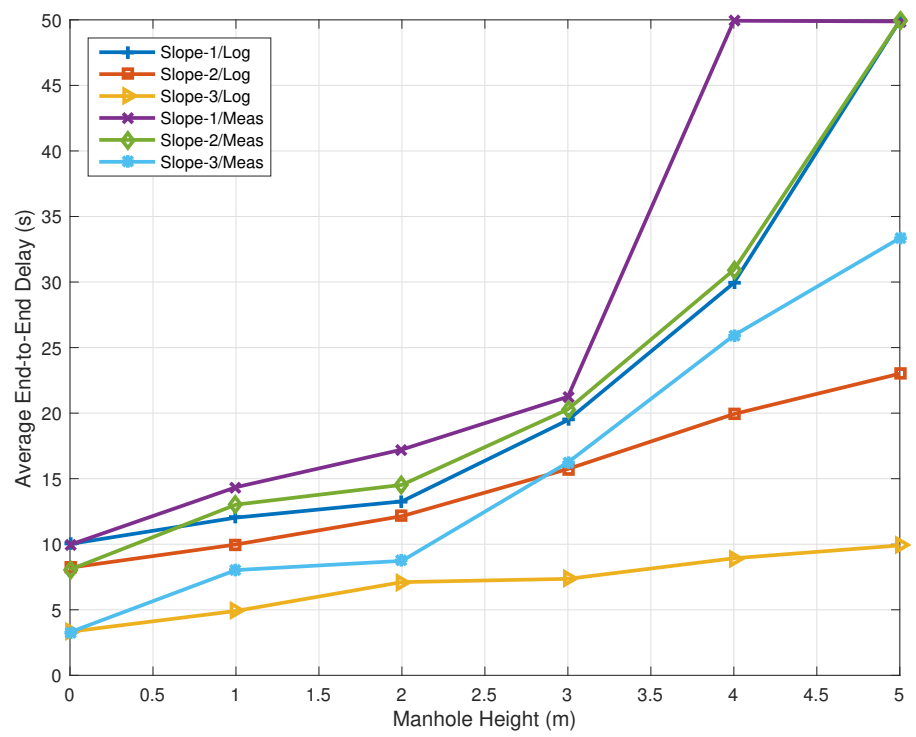

Fig. 7. End-to-end delay for no relaying mode with big tank size and with linear signal at various slopes. 


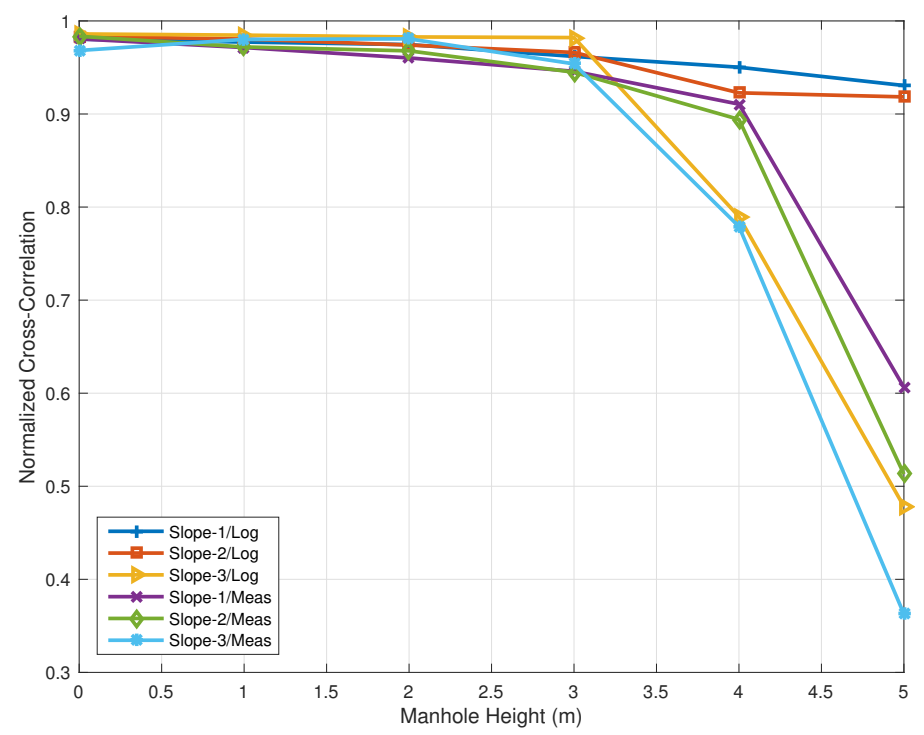

Fig. 8. Normalized cross-correlation for no relaying mode with big tank size and with linear signal at various slopes.

Table 1. Normalized absolute error for no relaying mode with big tank size and with charge signal at various time constants

\begin{tabular}{ccccccc}
\hline Manhole height $(\mathrm{m})$ & Log/Slope-1 & Log/Slope-2 & Log/Slope-3 & Meas/Slope-1 & Meas/Slope-2 & Meas/Slope-3 \\
\hline 0 & 0.00950 & 0.01222 & 0.01275 & 0.00955 & 0.01218 & 0.01274 \\
\hline 1 & 0.00992 & 0.01293 & 0.01324 & 0.01253 & 0.01598 & 0.01659 \\
\hline 2 & 0.01138 & 0.01401 & 0.01412 & 0.01537 & 0.02533 & 0.01717 \\
\hline 3 & 0.01992 & 0.01960 & 0.01579 & 0.02383 & 0.02897 & 0.01929 \\
\hline 4 & 0.02348 & 0.03023 & 0.01755 & 0.17749 & 0.03610 & 0.02615 \\
\hline 5 & 0.02965 & 0.04033 & 0.02498 & 0.44120 & 0.14791 & 0.04843 \\
\hline
\end{tabular}

Table 2. End-to-end delay in seconds for no relaying mode with big tank size and with charge signal at various time constants

\begin{tabular}{|c|c|c|c|c|c|c|}
\hline Manhole height (m) & Log/Slope-1 & Log/Slope-2 & Log/Slope-3 & Meas/Slope-1 & Meas/Slope-2 & Meas/Slope-3 \\
\hline 0 & 7.10 & 3.62 & 2.75 & 7.12 & 3.59 & 2.64 \\
\hline 1 & 9.05 & 6.02 & 4.05 & 13.14 & 6.78 & 5.27 \\
\hline 2 & 10.84 & 7.75 & 5.97 & 14.96 & 7.82 & 6.77 \\
\hline 3 & 20.67 & 20.73 & 11.61 & 21.24 & 18.18 & 14.04 \\
\hline 4 & 21.06 & 22.93 & 15.64 & 29.96 & 25.93 & 28.24 \\
\hline 5 & 23.89 & 25.31 & 49.91 & 49.96 & 31.94 & 49.91 \\
\hline
\end{tabular}


Table 3. Normalized cross-correlation for no relaying mode with big tank size and with charge signal at various time constants

\begin{tabular}{ccccccc}
\hline Manhole height (m) & Log/Slope-1 & Log/Slope-2 & Log/Slope-3 & Meas/Slope-1 & Meas/Slope-2 & Meas/Slope-3 \\
\hline 0 & 0.98453 & 0.98572 & 0.98602 & 0.98453 & 0.98580 & 0.98609 \\
\hline 1 & 0.98245 & 0.98427 & 0.98515 & 0.97800 & 0.98082 & 0.98441 \\
\hline 2 & 0.98007 & 0.98306 & 0.98402 & 0.97477 & 0.97662 & 0.98222 \\
\hline 3 & 0.96789 & 0.97534 & 0.98021 & 0.96495 & 0.97442 & 0.97557 \\
\hline 4 & 0.96693 & 0.94856 & 0.97710 & 0.78933 & 0.95341 & 0.94500 \\
\hline 5 & 0.96634 & 0.93688 & 0.96346 & 0.52371 & 0.84307 & 0.93918 \\
\hline
\end{tabular}

\subsection{Effect of Relaying}

In this subsection, we consider the importance of adding a relay at the manhole opening to improve connectivity due to the poor system performance, especially for large tanks. In both ideal and measured CIRs, adding the relay allows all the performance criteria to maintain almost the same performance as the LOS point-to-point communications at $D=0 \mathrm{~m}$, as shown in Figs. 9-11. A deeper look into the performance shown in Tables 4-15, demonstrates that there are very slight variations in the performance where the following behaviors are noticed.

The error and cross-correlation with relaying outperform the corresponding single node performance. On the contrary, the delay for the single node case is slightly lower because of the added delay due to two-hop transmissions. The change of delay is minor because the major delay component is the processing delay at the gateway. On all performance metrics, the performance degrades slightly with distance and by running the experiments using the measured CIR. The data for "Slope-1" and "Slope-3" were removed for brevity because similar behavior was captured in the performance metrics.

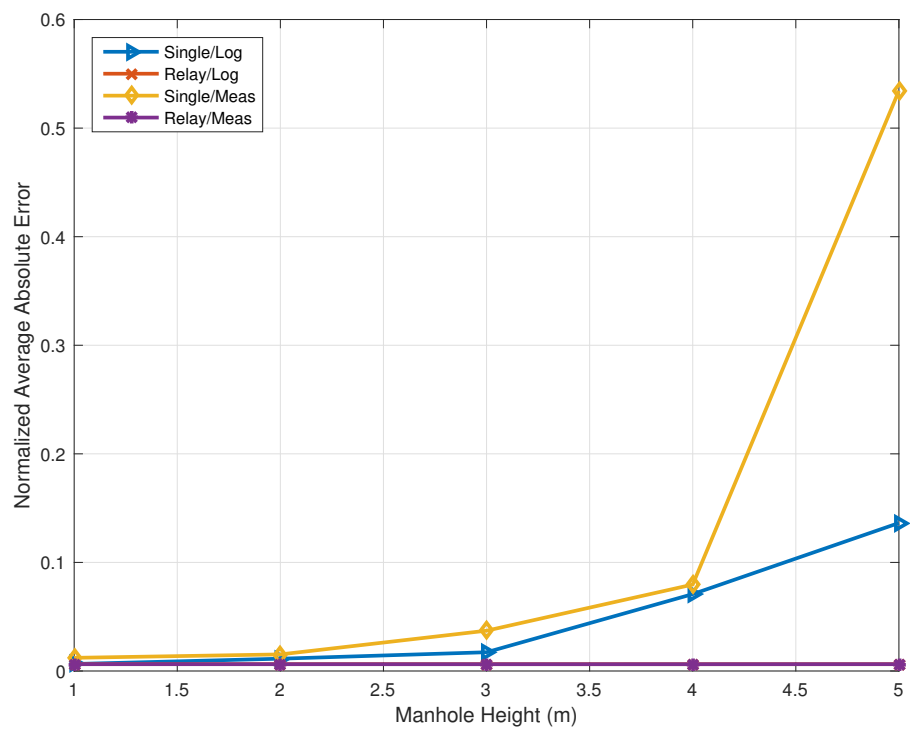

Fig. 9. Normalized absolute error with big tank size and with linear signal at $5 \mathrm{ppm} / \mathrm{min}$ slope. 


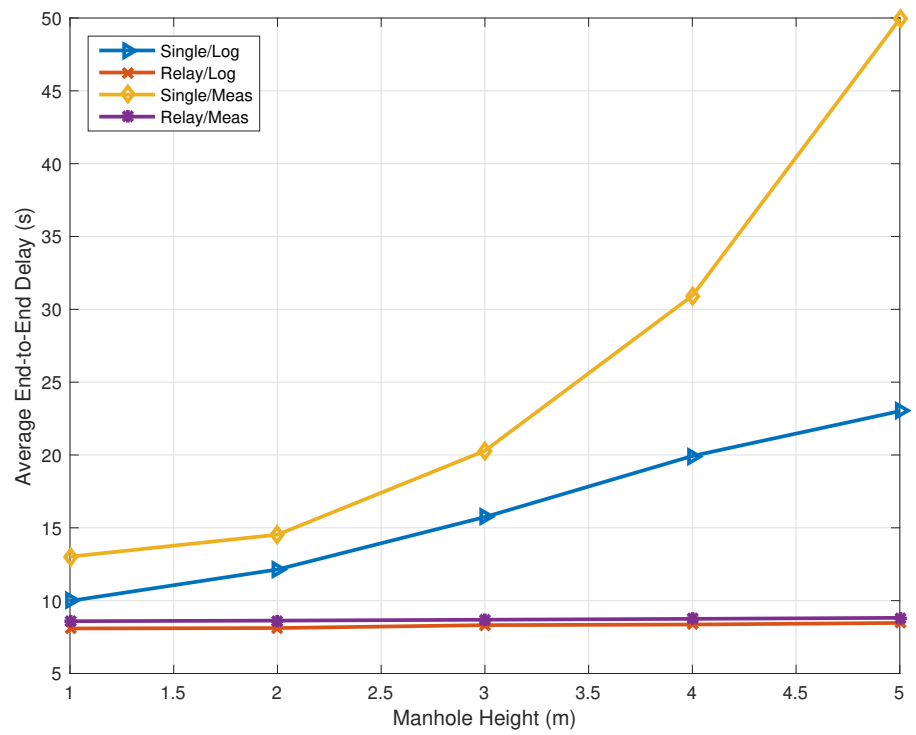

Fig. 10. End-to-end delay with big tank size and with linear signal at $5 \mathrm{ppm} / \mathrm{min}$ slope.

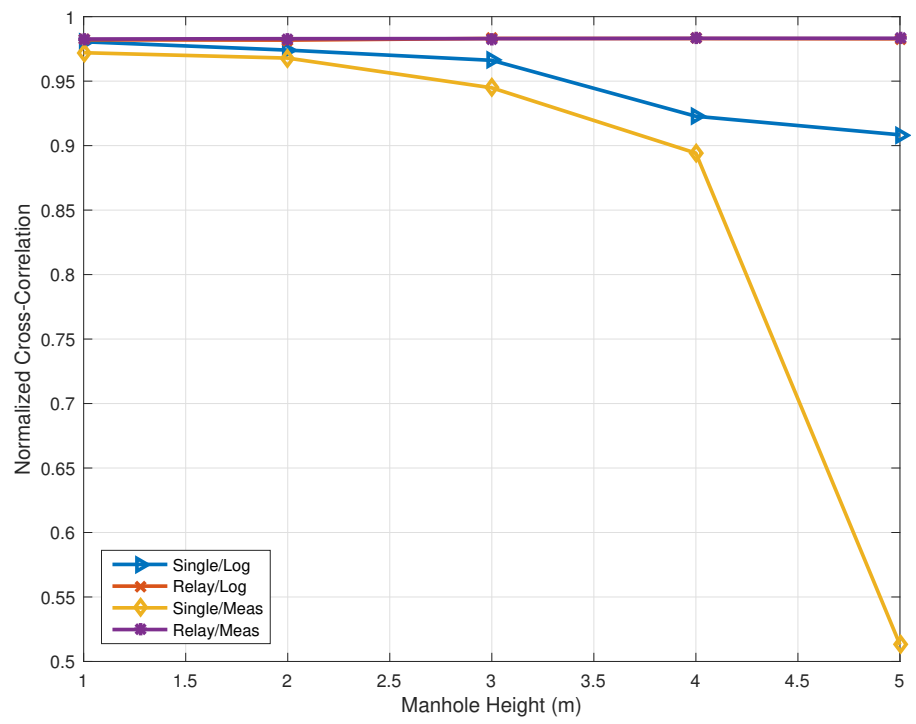

Fig. 11. Normalized cross-correlation with big tank size and with linear signal at $5 \mathrm{ppm} / \mathrm{min}$ slope.

Table 4. Normalized absolute error with big tank size and charge signal with 1.67 min time constant.

\begin{tabular}{ccccc}
\hline Manhole height $(\mathrm{m})$ & Single/Log & Relay/log & Single/Meas & Relay/Meas \\
\hline 1 & 0.01293 & 0.01220 & 0.01598 & 0.01218 \\
\hline 2 & 0.01401 & 0.01220 & 0.02533 & 0.01219 \\
\hline 3 & 0.01960 & 0.01223 & 0.02897 & 0.01224 \\
\hline 4 & 0.03023 & 0.01228 & 0.03610 & 0.01229 \\
\hline 5 & 0.04033 & 0.01230 & 0.14791 & 0.01236 \\
\hline
\end{tabular}


Table 5. End-to-end delay in seconds with big tank size and charge signal with 1.67 min time constant.

\begin{tabular}{|c|c|c|c|c|}
\hline Manhole height $(\mathrm{m})$ & Single/Log & Relay/log & Single/Meas & Relay/Meas \\
\hline 1 & 6.02 & 3.49 & 6.78 & 3.70 \\
\hline 2 & 7.75 & 3.59 & 7.82 & 3.81 \\
\hline 3 & 20.73 & 3.78 & 18.18 & 3.90 \\
\hline 4 & 22.93 & 3.82 & 25.93 & 4.33 \\
\hline 5 & 25.31 & 4.01 & 31.94 & 4.70 \\
\hline
\end{tabular}

Table 6. Normalized cross-correlation with big tank size and charge signal with 1.67 min time constant.

\begin{tabular}{ccccc}
\hline Manhole height (m) & Single/Log & Relay/log & Single/Meas & Relay/Meas \\
\hline 1 & 0.98427 & 0.98578 & 0.98082 & 0.98567 \\
\hline 2 & 0.98306 & 0.98560 & 0.97662 & 0.98553 \\
\hline 3 & 0.97534 & 0.98551 & 0.97442 & 0.98542 \\
\hline 4 & 0.94856 & 0.98542 & 0.95341 & 0.98538 \\
\hline 5 & 0.93688 & 0.98541 & 0.84307 & 0.98530 \\
\hline
\end{tabular}

Table 7. Normalized absolute error with big tank size and charge signal with 0.33 min time constant.

\begin{tabular}{ccccc}
\hline Manhole height $(\mathrm{m})$ & Single/Log & Relay/log & Single/Meas & Relay/Meas \\
\hline 1 & 0.01324 & 0.01274 & 0.01659 & 0.01274 \\
\hline 2 & 0.01412 & 0.01274 & 0.01717 & 0.01274 \\
\hline 3 & 0.01579 & 0.01280 & 0.01929 & 0.01288 \\
\hline 4 & 0.01755 & 0.01712 & 0.02615 & 0.01767 \\
\hline 5 & 0.02498 & 0.01873 & 0.04843 & 0.01976
\end{tabular}

Table 8. End-to-end delay in seconds with big tank size and charge signal with 0.33 min time constant.

\begin{tabular}{ccccc}
\hline Manhole height (m) & Single/Log & Relay/log & Single/Meas & Relay/Meas \\
\hline 1 & 4.05 & 2.51 & 5.27 & 2.63 \\
\hline 2 & 5.97 & 2.59 & 6.77 & 2.88 \\
\hline 3 & 11.61 & 2.88 & 14.04 & 2.98 \\
\hline 4 & 15.64 & 2.97 & 28.24 & 3.17 \\
\hline 5 & 49.91 & 3.07 & 49.91 & 3.74
\end{tabular}


Table 9. Normalized cross-correlation with big tank size and charge signal with 0.33 min time constant.

\begin{tabular}{ccccc}
\hline Manhole height (m) & Single/Log & Relay/log & Single/Meas & Relay/Meas \\
\hline 1 & 0.98515 & 0.98552 & 0.98441 & 0.98487 \\
\hline 2 & 0.98402 & 0.98549 & 0.98222 & 0.98486 \\
\hline 3 & 0.98021 & 0.98500 & 0.97557 & 0.98398 \\
\hline 4 & 0.97710 & 0.98352 & 0.94500 & 0.98301 \\
\hline 5 & 0.96346 & 0.98283 & 0.93918 & 0.98204 \\
\hline
\end{tabular}

Table 10. Normalized absolute error with small tank size and charge signal with 1.67 min time constant.

\begin{tabular}{ccccc}
\hline Manhole height $(\mathrm{m})$ & Single/Log & Relay/log & Single/Meas & Relay/Meas \\
\hline 1 & 0.01220 & 0.01212 & 0.01229 & 0.01218 \\
\hline 2 & 0.01223 & 0.01214 & 0.01230 & 0.01220 \\
\hline 3 & 0.01226 & 0.01214 & 0.01237 & 0.01221 \\
\hline 4 & 0.01229 & 0.01215 & 0.01246 & 0.01221 \\
\hline 5 & 0.01230 & 0.01225 & 0.01258 & 0.01221 \\
\hline
\end{tabular}

Table 11. End-to-end delay in seconds with small tank size and charge signal with 1.67 min time constant.

\begin{tabular}{|c|c|c|c|c|}
\hline Manhole height (m) & Single/Log & Relay/log & Single/Meas & Relay/Meas \\
\hline 1 & 3.52 & 4.40 & 3.77 & 4.42 \\
\hline 2 & 3.57 & 4.53 & 4.48 & 4.58 \\
\hline 3 & 3.59 & 4.56 & 4.57 & 4.65 \\
\hline 4 & 3.74 & 4.61 & 4.62 & 4.67 \\
\hline 5 & 3.78 & 4.63 & 5.58 & 4.70 \\
\hline
\end{tabular}

Table 12. Normalized cross-correlation with small tank size and charge signal with 1.67 min time constant.

\begin{tabular}{ccccc}
\hline Manhole height (m) & Single/Log & Relay/log & Single/Meas & Relay/Meas \\
\hline 1 & 0.98598 & 0.98684 & 0.98564 & 0.98638 \\
\hline 2 & 0.98595 & 0.98676 & 0.98520 & 0.98630 \\
\hline 3 & 0.98590 & 0.98630 & 0.98515 & 0.98621 \\
\hline 4 & 0.98589 & 0.98623 & 0.98514 & 0.98614 \\
\hline 5 & 0.98573 & 0.98623 & 0.98452 & 0.98614 \\
\hline
\end{tabular}


Table 13. Normalized absolute error with small tank size and linear signal with $5 \mathrm{ppm} / \mathrm{min}$ slope.

\begin{tabular}{ccccc}
\hline Manhole height (m) & Single/Log & Relay/log & Single/Meas & Relay/Meas \\
\hline 1 & 0.00631 & 0.00624 & 0.00631 & 0.00626 \\
\hline 2 & 0.00631 & 0.00626 & 0.00632 & 0.00628 \\
\hline 3 & 0.00633 & 0.00628 & 0.00636 & 0.00630 \\
\hline 4 & 0.00635 & 0.00629 & 0.00642 & 0.00632 \\
\hline 5 & 0.00636 & 0.00630 & 0.00646 & 0.00632 \\
\hline
\end{tabular}

Table 14. End-to-end delay in seconds with small tank size and linear signal with $5 \mathrm{ppm} / \mathrm{min}$ slope.

\begin{tabular}{ccccc}
\hline Manhole height (m) & Single/Log & Relay/log & Single/Meas & Relay/Meas \\
\hline 1 & 8.02 & 7.91 & 8.14 & 8.29 \\
\hline 2 & 8.06 & 8.13 & 8.95 & 8.39 \\
\hline 3 & 8.07 & 8.57 & 8.98 & 8.51 \\
\hline 4 & 8.10 & 8.77 & 9.37 & 8.60 \\
\hline 5 & 8.12 & 8.91 & 9.72 & 8.68 \\
\hline
\end{tabular}

Table 15. Normalized cross- correlation with small tank size and linear signal with $5 \mathrm{ppm} / \mathrm{min}$ slope.

\begin{tabular}{|c|c|c|c|c|}
\hline Manhole height (m) & Single/Log & Relay/log & Single/Meas & Relay/Meas \\
\hline 1 & 0.98382 & 0.98416 & 0.98343 & 0.98346 \\
\hline 2 & 0.98356 & 0.98389 & 0.98196 & 0.98332 \\
\hline 3 & 0.98354 & 0.98388 & 0.98174 & 0.98276 \\
\hline 4 & 0.98350 & 0.98367 & 0.98107 & 0.98262 \\
\hline 5 & 0.98341 & 0.98358 & 0.98093 & 0.98249 \\
\hline
\end{tabular}

\subsection{Effect of Humans in the Confined Space}

Finally, we consider the effect of having absorbers inside the confined space. These absorbers can practically represent workers inside the tank performing some task, such as welding, which may produce various types of gases. The performance metrics with one and two workers were compared to the benchmark system of an empty tank. In all cases, relaying was considered.

In Figs. 12-14, the performance degrades for the case of two workers. However, the performance is still much better compared to the single node scenario. The assumption of fixed attenuation makes the performance have minor variations against the manhole height.

In Tables 16-18, similar behavior is shown for both small and big tanks, and linear and charge input signals. Having more absorbers inside a confined space may degrade the performance significantly, and hence more relays can be added. Furthermore, ISA100.11a sensor nodes have relaying capabilities, and so distributed sensor networks can be beneficial for achieving the required connectivity in confined spaces. The data for "Slope-1" and "Slope-3" were removed for brevity because similar behavior was noticed in the performance. 


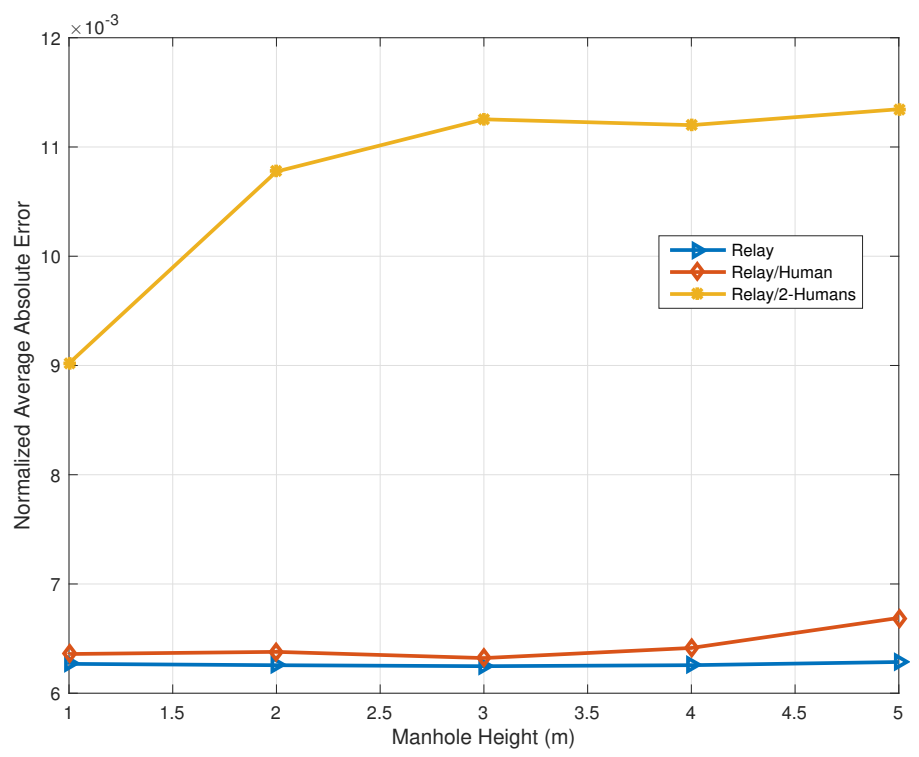

Fig. 12. Normalized absolute error with big tank size and with linear signal at $5 \mathrm{ppm} / \mathrm{min}$ slope and measured channel CIR.

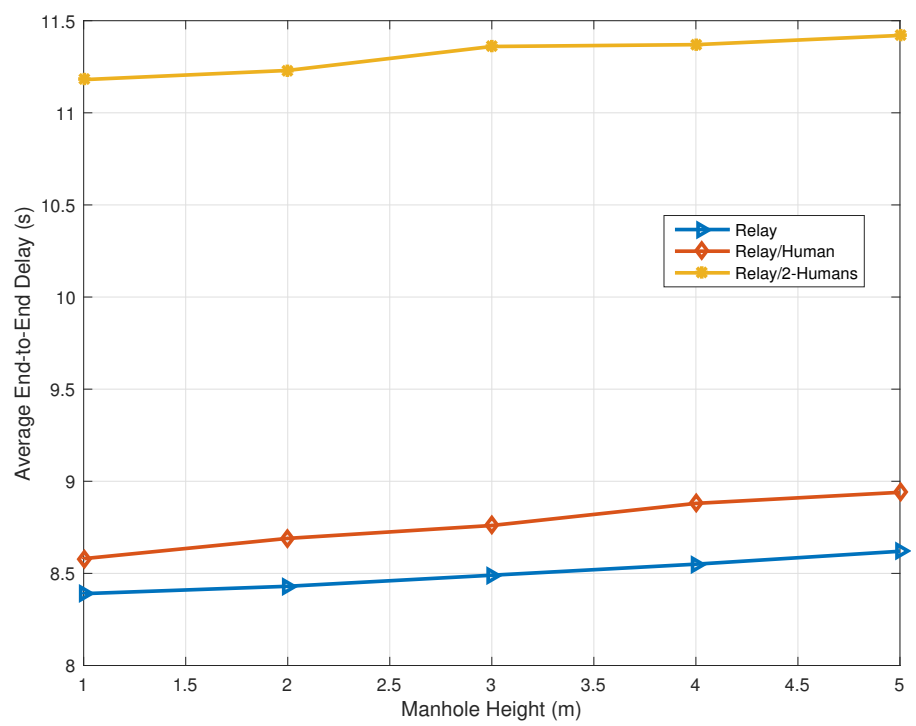

Fig. 13. End-to-end delay with big tank size and with linear signal at $5 \mathrm{ppm} / \mathrm{min}$ slope and measured channel CIR. 


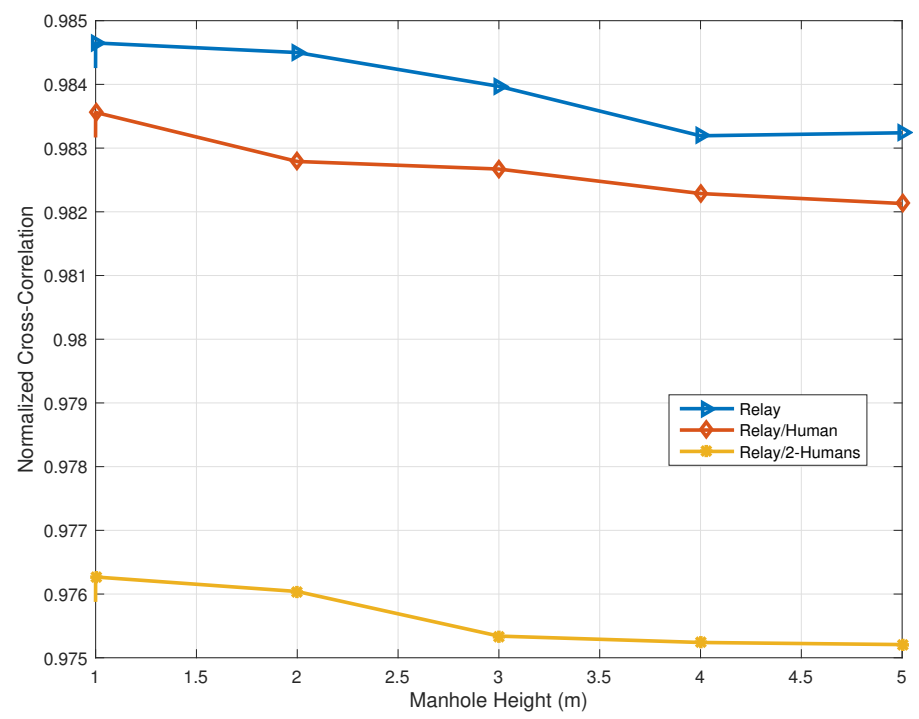

Fig. 14. Normalized cross-correlation with big tank size and with linear signal at $5 \mathrm{ppm} / \mathrm{min}$ slope and measured channel CIR.

Table 16. Normalized absolute error with big tank size and charge signal with 1.67 min time constant and measured channel CIR.

\begin{tabular}{cccc}
\hline Manhole height $(\mathrm{m})$ & No Human & 1 Human & 2 Humans \\
\hline 1 & 0.01218 & 0.01233 & 0.01333 \\
\hline 2 & 0.01219 & 0.01238 & 0.01310 \\
\hline 3 & 0.01224 & 0.01243 & 0.01436 \\
\hline 4 & 0.01229 & 0.01243 & 0.01462 \\
\hline 5 & 0.01236 & 0.01246 & 0.02016 \\
\hline
\end{tabular}

Table 17. End-to-end delay in seconds with big tank size and charge signal with 1.67 min time constant and measured channel CIR.

\begin{tabular}{cccc}
\hline Manhole height $(\mathrm{m})$ & No Human & 1 Human & 2 Humans \\
\hline 1 & 3.70 & 3.83 & 5.32 \\
\hline 2 & 3.81 & 3.92 & 5.62 \\
\hline 3 & 3.90 & 3.99 & 7.12 \\
\hline 4 & 4.33 & 4.37 & 7.29 \\
\hline 5 & 4.70 & 4.82 & 7.56 \\
\hline
\end{tabular}


Table 18. Normalized cross-correlation with big tank size and charge signal at 1.67 min time constant and measured channel CIR.

\begin{tabular}{cccc}
\hline Manhole height $(\mathrm{m})$ & No Human & 1 Human & 2 Humans \\
\hline 1 & 0.98567 & 0.98469 & 0.98347 \\
\hline 2 & 0.98553 & 0.98450 & 0.98286 \\
\hline 3 & 0.98542 & 0.98442 & 0.98280 \\
\hline 4 & 0.98538 & 0.98414 & 0.98276 \\
\hline 5 & 0.98530 & 0.98409 & 0.97865 \\
\hline
\end{tabular}

\section{Conclusions}

In this paper, we studied the performance of industrial wireless networks operating in a confined space, where the use case of gas sensing was considered. We employed an HIL test bed where the measured CIR of a confined space is used through a channel emulator. By building such an industrial test bed with an ISA100.11a-based wireless sensor network and a channel emulator to mimic various industrial environmental effects, we were able to study the performance of an industrial wireless network in a realistic setting. We considered three end-to-end performance criteria, namely, the normalized average absolute error, the average delay, and normalized peak cross-correlation. Practical input signals were injected to the gas sensor, and the received signal characteristics were evaluated.

First, we have shown that the single sensor case can have a satisfactory performance for smaller confined spaces with or without the existence of LOS. Relaying is needed for NLOS scenarios with longer distances between the sensor and the gateway. Relaying was able to achieve a very close performance to the LOS single-link case. However, the relaying can add negligible end-to-end delay for two-hop networks. This delay is expected to become worse with an increase in the number of hops. We have shown the robustness of the tested system for various input signal rates. Finally, in the case where workers (absorbers) exist inside a confined space close to the sensor node, path redundancy may be required to overcome the attenuation of the transmitted signals.

\section{References}

[1] Liu Y, Candell R, Lee K, Moayeri N (2016) A simulation framework for industrial wireless networks and process control systems. 2016 IEEE World Conference on Factory Communication Systems (WFCS), pp 1-11. https://doi.org/10.1109/WFCS.2016.7496495

[2] Candell R, Kashef M (2017) Industrial wireless: Problem space, success considerations, technologies, and future direction. 2017 Resilience Week (RWS), pp 133-139. https://doi.org/10.1109/RWEEK.2017.8088661

[3] Electronics Design (2013) Connectivity provides the lifeline of industrial control. Available at http://electronicdesign.com/industrial/connectivity-provides-lifeline-industrial-control

[4] GE (2016) Industrial communications professional services: Delivering high-performing communication network systems for industrial applications. Available at https://www.gegridsolutions.com/Communications/ProfessionalServices_GEA-12702B-E_160418_R001.pdf

[5] Smith D, Veitch B, Khan F, Taylor R (2017) Understanding industrial safety: Comparing fault tree, bayesian network, and \{FRAM\} approaches. Journal of Loss Prevention in the Process Industries 45:88-101. https://doi.org/http://doi.org/10.1016/j.jlp.2016.11.016

[6] Chraim F, Erol YB, Pister K (2016) Wireless gas leak detection and localization. IEEE Transactions on Industrial Informatics 12(2):768-779. https://doi.org/10.1109/TII.2015.2397879

[7] Huber A, Weiss A (2017) Developing human-robot interaction for an industry 4.0 robot: How industry workers helped to improve remote-HRI to physical-HRI. Proceedings of the Companion of the 2017 ACM/IEEE International Conference on Human-Robot Interaction, pp 137-138. https://doi.org/10.1145/3029798.3038346 
[8] Zanchettin AM, Ceriani NM, Rocco P, Ding H, Matthias B (2016) Safety in human-robot collaborative manufacturing environments: Metrics and control. IEEE Transactions on Automation Science and Engineering 13(2):882-893. https://doi.org/10.1109/TASE.2015.2412256

[9] Workplace Safety and Health Council (2008) Technical advisory on working safely in confined spaces. Available at https://www.wshc.sg/files/wshc/upload/cms/file/2014/\cs2.pdf

[10] Zhang L, Gao H, Kaynak O (2013) Network-induced constraints in networked control systems: A survey. IEEE Transactions on Industrial Informatics 9(1):403-416. https://doi.org/10.1109/TII.2012.2219540

[11] Neema H, Gohl J, Lattmann Z, Sztipanovits J, Karsai G, Neema S, Bapty T, Batteh J, Tummescheit H, Sureshkumar C (2014) Model-based integration platform for FMI co-simulation and heterogeneous simulations of cyber-physical systems. Proceedings of the 10th International Modelica Conference, pp 235-245.

[12] Bause F, Buchholz P, Kriege J, Vastag S (2010) A simulation environment for hierarchical process chains based on OMNeT++. Simulation 86(5-6):291-309. https://doi.org/10.1177/0037549709104236

[13] Rezha FP, Shin SY (2013) Performance evaluation of ISA100.11A industrial wireless network. IET International Conference on Information and Communications Technologies (IETICT 2013), pp 587-592. https://doi.org/10.1049/cp.2013.0105

[14] Han S, Zhu X, Mok AK, Chen D, Nixon M (2011) Reliable and real-time communication in industrial wireless mesh networks. 2011 17th IEEE Real-Time and Embedded Technology and Applications Symposium, pp 3-12. https://doi.org/10.1109/RTAS.2011.9

[15] Han S, Song J, Zhu X, Mok AK, Chen D, Nixon M, Pratt W, Gondhelekar V (2009) Wi-HTest: Compliance test suite for diagnosing devices in real-time WirelessHART network. 2009 15th IEEE Real-Time and Embedded Technology and Applications Symposium, pp 327-336. https://doi.org/10.1109/RTAS.2009.18

[16] Wu L, Xu S, Jiang D (2015) MFAHP: A novel method on the performance evaluation of the industrial wireless networked control system. Proceedings of the Sixth International Conference on Electronics and Information Engineering, Volume 9794, p 97940E. https://doi.org/10.1117/12.2203479

[17] Wu LQ, Xu S (2014) Research on the performance test of wired/wireless heterogeneous industrial control network. Applied Mechanics and Materials 599-601:1665-1670. https://doi.org/10.4028/www.scientific.net/AMM.599-601.1665

[18] Li T, Fei M, Hu H (2010) Performance analysis of industrial wireless network based on IEEE 802.15.4a (Springer, Berlin, Heidelberg), pp 64-69. https://doi.org/10.1007/978-3-642-15853-7_9

[19] Li TT, Jia TG, Fei MR, Hu HS (2011) Time delay characteristic of industrial wireless networks based on IEEE 802.15.4a. International Journal of Automation and Computing 8(2):170. https://doi.org/10.1007/s11633-011-0570-8

[20] Zheng M, Liang W, Yu H, Xiao Y (2015) Performance analysis of the industrial wireless networks standard: WIA-PA. Mobile Networks and Applications 22(1):139-150.https://doi.org/10.1007/s11036-015-0647-7

[21] Cena G, Bertolotti IC, Valenzano A, Zunino C (2007) Evaluation of response times in industrial WLANs. IEEE Transactions on Industrial Informatics 3(3):191-201. https://doi.org/10.1109/TII.2007.903219

[22] Ferrari G, Medagliani P, Di Piazza S, Martalò M (2007) Wireless sensor networks: Performance analysis in indoor scenarios. EURASIP Journal on Wireless Communications and Networking 2007(1):081864. https://doi.org/10.1155/2007/81864

[23] Hunter GW, Stetter JR, Hesketh P, Liu CC (2010) Smart sensor systems. The Electrochemical Society Interface 19(4):29-34. https://doi.org/10.1149/2.F03104if

[24] Leccardi M, et alDecarli M, Lorenzelli L, Milani P, Orava R, Barborini E (2012) Long-term outdoor reliability assessment of a wireless unit for air-quality monitoring based on nanostructured films integrated on micromachined platforms. Sensors 12(12):8176-8192. https://doi.org/10.3390/s120608176

[25] Somov A, Baranov A, Spirjakin D (2014) A wireless sensor-actuator system for hazardous gases detection and control. Sensors and Actuators A: Physical 210:157 - 164. https://doi.org/http://dx.doi.org/10.1016/j.sna.2014.02.025

[26] Jain PC, Kushwaha R (2012) Wireless gas sensor network for detection and monitoring of harmful gases in utility areas and industries. 2012 Sixth International Conference on Sensing Technology (ICST), pp 642-646. https://doi.org/10.1109/ICSensT.2012.6461759

[27] Abu-Mahfouz AM, Isaac SJ, Kruger CP, Aakvaag N, Fismen B (2014) Wireless gas sensing in south african underground platinum mines. 2014 IEEE Wireless Communications and Networking Conference (WCNC), pp 3432-3437. https://doi.org/10.1109/WCNC.2014.6953135

[28] AFSCME (2011) Health and safety factsheet: Welding hazards. Available at http: //www.afscme.org/news/publications/workplace-health-and-safety/fact-sheets/pdf/Welding-Hazards-AFSCME-fact-sheet.pdf

[29] TWI(ND) What are the hazards from gases during welding and cutting? Available at http://www.twi-global.com/technical-knowledge/faqs/faq-what-are-the-hazards-from-gases-during-welding-and-cutting/

[30] Antonsson AB, Christensson B, Berge J, Sjögren B (2013) Fatal carbon monoxide intoxication after acetylene gas welding of pipes. The Annals of Occupational Hygiene 57(5):662-666. https://doi.org/10.1093/annhyg/mes104

[31] RFnest (2017) Product specifications. Available at http://www.i-a-i.com/wp-content/uploads/2017/07/RFnest-Specsheet-2017.pdf

[32] Mehlführer C, Rupp M (2008) Approximation and resampling of tapped delay line channel models with guaranteed channel properties. 2008 IEEE International Conference on Acoustics, Speech and Signal Processing, pp 2869-2872. 
https://doi.org/10.1109/ICASSP.2008.4518248

[33] Taha-Ahmed B, Alvarez Calvo I, Masa Campos J (2013) Materials' insertion loss at 2.4, 3.3 and 5.5ghz bands. Progress In Electromagnetics Research 30:1-10. https://doi.org/10.2528/PIERM13022803

\begin{abstract}
About the authors: Mohamed Kashef is a guest research associate in the Advanced Network Technologies Division at NIST.

Richard Candell is an electronics engineer in the Intelligent Systems Division at NIST. He leads a research project in wireless networks used for industrial control applications.

The National Institute of Standards and Technology is an agency of the U.S. Department of Commerce.
\end{abstract}

